\title{
Decoding mechanism of plastid division: structure and kinetics of the plastid-dividing machinery
}

\author{
Yamato Yoshida \\ Laboratory for Single Cell Gene Dynamics, Quantitative Biology Center, RIKEN, Furuedai, Suita, Osaka 565-0874, Japan \\ Author for correspondence: Y. Yoshida, yamato.yoshida@riken.jp
}

Summary: Chloroplasts (plastids) are able to synthesize energy-containing macromolecules for a great deal of living organisms. Consistent with their endosymbiotic origin, plastids maintain themselves by binary division. Plastid division is carried out by a ring complex called the plastid-dividing (PD) machinery; the PD machinery has inner and outer ring structures across the plastid membranes. Although many studies have been done to reveal the mechanisms of plastid division, much about the components and molecular mechanisms of the PD machinery remain to be discovered. My work demonstrated that: (1) the contractile force of the PD ring is generated via filament-sliding movement by dynamin proteins; (2) the PD ring is composed of polyglucan nanofilaments, synthesized by the glucosyltransferase PDR1; and (3) examination of the FtsZ ring reconstituted in a heterologous system revealed the assembly and contractile dynamics of the FtsZ ring. In addition, we have recently established isolation of the mitochondriondividing (MD) machinery and revealed that the ultrastructure and the dynamics of the isolated MD machinery were similar to those of the isolated PD machinery. Therefore, plastids and mitochondria divide by the action of supramolecular complexes "the PD and MD machineries" including dual contractible rings, the PD/MD ring and the FtsZ ring. These findings will lead to an understanding of how plastids and mitochondria were established during evolution.

Key words: Cyanidioschyzon merolae, dynamin, FtsZ, PD ring, plastid division

\section{INTRODUCTION}

Plastids evolved from a cyanobacterial ancestor by endosymbiosis. Due to this reason, plastids have retained their genome system and increase multiplicatively via division, which is mediated by a duplex ring complex called the plastid-dividing (PD) machinery (Kuroiwa et al. 2008, Miyagishima et al. 2011, Yoshida et al. 2012, Jarvis and López-Juez 2013, Osteryoung and Pyke 2014). The PD machinery includes inner and outer ring structures that assemble at the plastid division site and cleave the plastid into two daughter plastids. During plastid division, the FtsZ ring assembles on the stromal side of the plastid inner envelope membrane as the inner ring of the PD machinery (Mori et al. 2001, Vitha et al. 2001), and the PD ring, a bundle of nanofilaments, assembles on the cytosolic side of the outer envelope membrane as the outer ring (Kuroiwa et al. 1998, Miyagishima et al. 2001). A dynamin-related GTPase (known as Dnm2 in Cyanidioschyzon merolae and DRP5B or ARC5 in Arabidopsis thaliana) also participates in plastid division as part of the outer PD machinery (Gao et al. 2003, Miyagishima et al. 2003). To uncover the mechanisms of plastid division, we used the primitive alga C. merolae (Kuroiwa 1998). Containing just one nucleus, one mitochondrion, and one plastid, divisions of which can be highly synchronized, C. merolae offers striking unique advantages for studies of plastid and mitochondrial divisions (Figure 1A and 1B). Also, simple genome structure and gene system of C. merolae enable various types of omics approaches (Matsuzaki et al. 2004, Misumi et al. 2005, Nozaki et al. 2007, Fujiwara et al. 2009, Yoshida et al. 2011). Emerging work has established antisense suppression (Ohnuma et al. 2009), inducible gene expression (Sumiya et al. 2014), and conditional gene knockdown systems for C. merolae (Minoda et al. 2004, Fujiwara et al. 2015, Fujiwara et al. 2017). During a last decade, I have studied structural detail and molecular mechanism of the PD machinery by using isolated PD machineries from C. merolae cells (Yoshida et al. 2006, 2010). Furthermore, I recently established synthetic biological approaches to study molecular mechanisms and assembly dynamics of the FtsZ ring (Yoshida and Mogi 2016, Yoshida et al. 2016). Here, I introduce the plastid division mechanism executed by the PD machinery.

\section{STRUCTURE AND DYNAMICS OF ISOLATED PD MACHINERIES}

Plastid division is executed by the PD machinery that includes a chimera of the rings originated from bacteria (FtsZ ring) and eukaryotes (PD and dynamin rings), but the role of each ring in the PD machinery is unclear. Therefore, I attempted to isolate the PD machinery from the cells of the unicellular red alga $C$. merolae, which contains a single plastid per cell with a robust PD machinery. To isolate PD machineries, dividing plastids were obtained from synchronized cells and treated with a detergent cocktail of Nonidet P-40 and $n$-octyl- $\beta$-D-glucopyranoside. Finally, I have established isolation method of the intact PD machineries from C. merolae. Interestingly, isolated PD machineries formed super-twisted rings and spirals in addition to circular rings (Figures 1C-E). Also, the spiral type of isolated PD machineries took both clockwise and counter-clockwise form (Figure 1D). When the plastid membranes were dissolved by the detergent, a membrane-free PD machinery autonomously constricted. The existence of these twisted structures and the autonomous transformation property indicated that the PD machineries generate the contractile force for plastid division, thereby posing the question of which protein components in the PD machineries generate the force. Although the addition of guanosine triphosphate (GTP) did not stimulate any significant conformational changes, longer detergent treatment produced FtsZ- or dynamin-released PD machineries. Interestingly, the 
FtsZ-released PD machineries could maintain spiral structures, whereas many of the dynamin-released PD machineries were straight (Figure 1F). As these facts seemed to indicate an importance of dynamin proteins in the contractile mechanism of the PD machinery, we examined optical trapping and manipulation experiments using optical tweezers to further elucidate it. When individual spiral PD machineries were stretched to four times their original lengths by optical laser trapping, they returned to their original sizes upon release. However, the plasticity of the spiral was lost after several stretches. Similar results were obtained for many spiral PD machineries. In contrast, dynamin-released straight PD machineries were unable to recover from stretch. Thus, a series

A
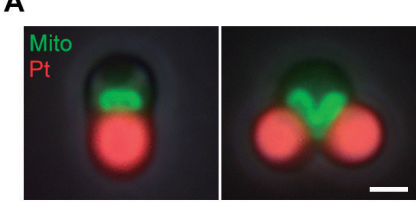

B

C
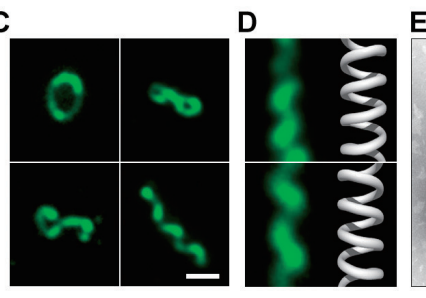

E
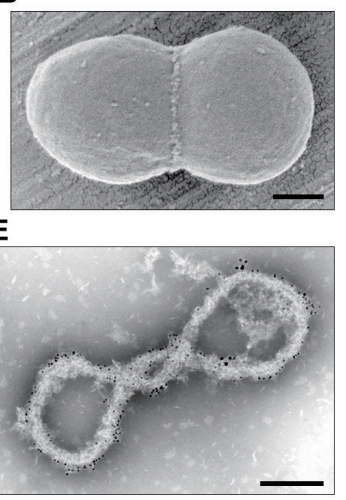

$\mathrm{F}$
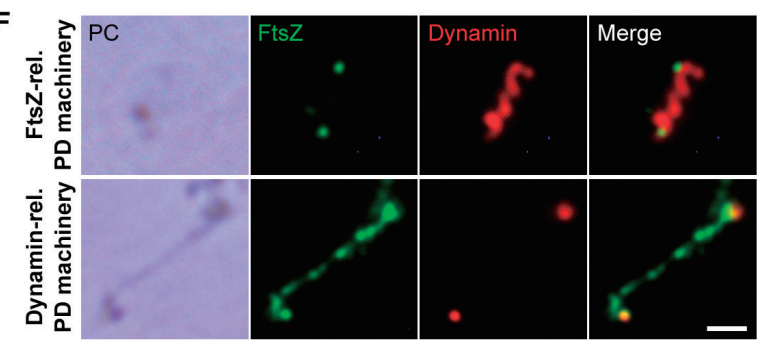

G

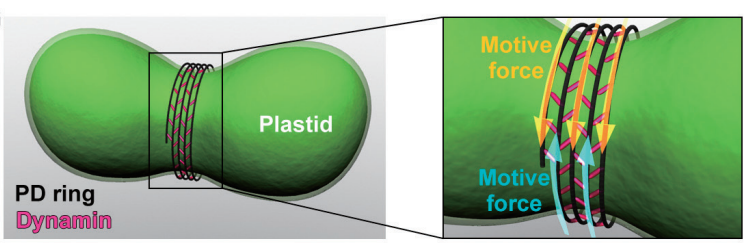

Figure 1 Structure and dynamics of the isolated PD machineries. (A) Cell structure of unicellular red alga Cyanidioschyzon merolae. Mitochondria (Mito, green) were immunolabeled using an anti-mitochondrial porin antibody, and plastid ( $\mathrm{Pt}$, red) emitted red autofluorescence. Bar $=1$ $\mu \mathrm{m}$. (B) A scanning electron-microscopic image of an isolated dividing plastid. The PD machinery is assembled at the division site of a plastid. Bar $=500 \mathrm{~nm}$. (C) Isolated PD machineries form following three-types of structures; circular form, super-twisted form, and spiral form. Bar $=1 \mu \mathrm{m}$. (D) Spiral PD machineries can form either clockwise or counter-clockwise rotation. (E) An immunoelectron microscopic image of a super-twisted PD machinery. Bar $=200 \mathrm{~nm}$. (F) Partial-disassemble assays of isolated PD machineries by CHAPS detergent treatment. Isolated PD machineries were immunolabeled using anti-FtsZ2 antibody (green) and anti-Dnm2 antibody (red). Bar $=1 \mu \mathrm{m}$. (G) Schematic representations of plastid division by the PD machinery. PD ring filaments were seemingly slid by cross-linking dynamin (Dnm2) molecules to generate the motive force (orange and blue arrows) for constriction of plastid division site. Images were modified from Yoshida et al. (2006) and Yoshida et al. (2010). of results indicates that dynamin generates the motive force for contraction (Yoshida et al. 2006).

To elucidate the kinetic dynamics of the PD, dynamin, and FtsZ rings in the PD machineries, we examined nanoscale observation of isolated PD machineries. A bundle of PD-ring nanofilaments was clearly observed and a single PD-ring filament had an average width of 5-7 nm. FtsZ signals were located on the inner periphery of the circular PD ring where the membrane was dissolved. On the other hand, Dynamin proteins were located in spiral line along the outer periphery of the supertwisted and spiral PD machineries (Figure 1E). Particularly, dynamin signals were identified between the PD-ring filaments, suggesting that the dynamin molecules crosslink with the PD ring filaments to generate the motive force for contraction by the sliding-movement of the PD ring filaments (Figure 1G) (Yoshida et al. 2006).

\section{ELUCIDATION OF MOLECULAR STRUCTURE AND ASSEMBLY MECHANISM OF THE PD RING}

The PD ring is a main skeletal structure of the PD machinery and is a universal structure in the plant kingdom (Kuroiwa et al. 1998). However, the molecular components and assembly of the PD ring have been enigmatic. To elucidate the components of the PD ring, we digested isolated PD machineries with various proteases but could not decompose them. In addition, electron-dense deposits indicating carbohydrates appeared on the PD ring after staining with periodic acid (PA)-horseradish peroxidase (HRP). The results suggested that the PD ring is likely composed of a saccharic architecture. Based on this finding, we next attempted to find out hypothetical protein components that might constitute the PD ring and relate with saccharide molecules. For the purpose, we took multi-omics approach. We initially isolated PD machineries from dividing C. merolae cells and subjected to proteomic analysis using matrix-assisted laser desorption/ionization-time-of-flight mass spectrometry (MALDI-TOF-MS). By the proteomic analysis, a glycogenin-like protein, CMR358C, which is part of a subgroup of glycosyltransferase proteins, was identified and designated as PLASTID-DIVIDING RING 1 (PDR1). A characteristic feature of PDR1 is a putative glycosyltransferase domain in the C-terminal region. Homologs of PDR1 are identified in various photosynthetic eukaryotes including red alga and land plants. Then, transcriptomic analyses showed that the mRNA and protein levels of PDR1 increased during the S to M phase, which coincides with the plastid division phase in C. merolae. Immunofluorescence microscopy demonstrated that PDR1 appeared earlier than dynamin in the cytoplasm and formed a ring from the beginning to the end of plastid division (Figure 2A). Furthermore, antisense-suppression of $P D R 1$ significantly decreased the frequency of plastid division compared with control cells, suggesting that PDR1 is essential for plastid division. Thus, we concluded that PDR1 is a novel plastid division protein.

To investigate the relationship between PDR1 and PD ring filaments, we performed immunoelectron microscopy using 
isolated PD machineries (Figure 2B). PDR1 was localized uniformly on the PD machineries, suggesting that PDR1 is a fundamental and/or structural component of the PD machinery. Particularly, many more PDR1 proteins were detected in the less-condensed region of PD ring filaments than in the solid region (Figure 2B, insets), suggesting that PDR1 proteins are associated with the whole of the PD ring filament. To investigate whether PDR1 proteins bind to saccharide molecules, we next examined a glycoprotein-detection analysis using the isolated PD machineries. As a result, a strong fluorometric signal was detected in the fraction and was confirmed to derive from PDR1 by MALDI-TOF-MS. In addition, many nanofilaments remained after removal of proteins from isolated PD machineries by protease and ionic treatments, and componential analyses revealed that the filamentary fraction contained only glucose

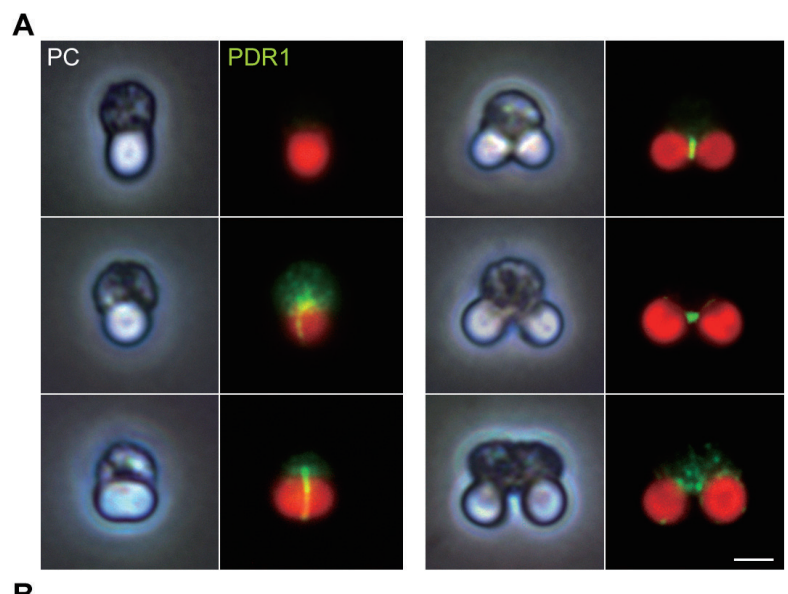

B
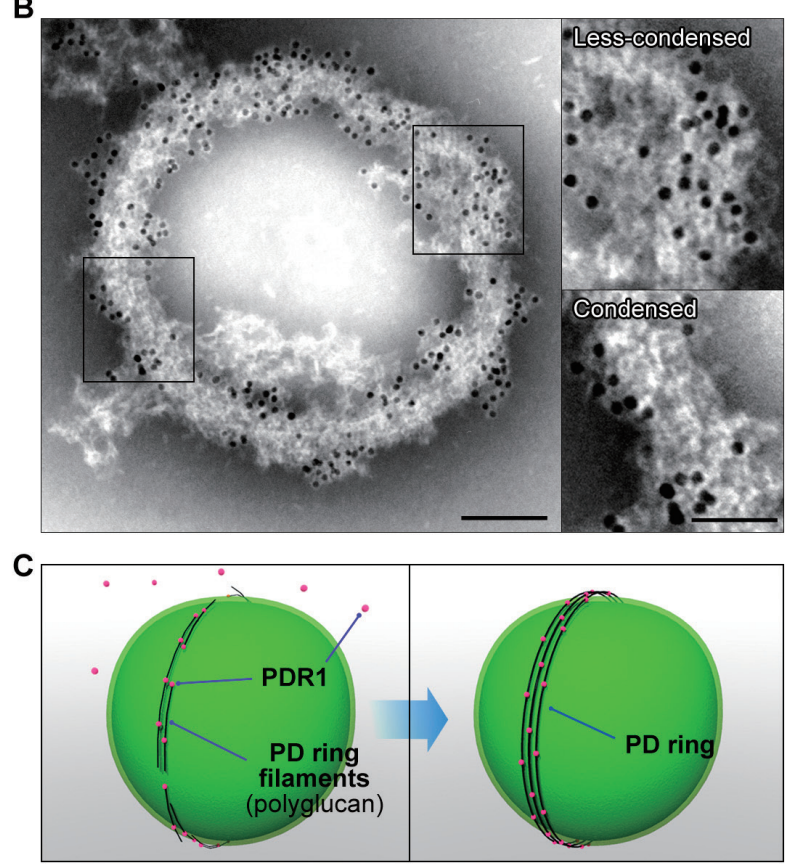

Figure 2 Assembly of the PD ring by PLASTID-DIVIDING RING1 (PDR1). (A) Intracellular dynamics of PDR1 proteins during plastid division. Bar $=1 \mu \mathrm{m}$. (B) Immunoelectron microsocopic images of an isolated PD machinery immunolabeled using anti-PDR1 antibody. Bars = $100 \mathrm{~nm}$ (left) and $50 \mathrm{~nm}$ (right). (C) A working model of biosynthesis of PD ring filaments by PDR1 proteins and glucose molecules. Images were modified from Yoshida et al. (2010). molecules. Taken together, the PD ring filaments are composed of PDR1-mediated polyglucan chains. PDR1 has sequence similarity to glycogenin, which play as a priming protein for glycogen synthesis; therefore, it is now hypothesized that PDR1 can also elongate the glucan chain to synthesize PD ring filaments, analogous to the synthesis of glycogen (Figure 2C) (Yoshida et al. 2010).

\section{MOLECULAR EVOLUTION OF A BACTERIA-DERIVED DIVISION SYSTEM}

Plastid FtsZs are tubulin-like GTPases that descended from a precursor in the cyanobacterial ancestor of plastids. Interestingly, whereas bacteria have one FtsZ gene in their genomes, the plastid FtsZ gene was duplicated and these duplicated loci, now present in the nuclear genomes, are widely conserved throughout photosynthetic eukaryotes (Miyagishima et al. 2004, TerBush et al. 2013). Phylogenetic studies classified the plastid FtsZs into two groups: FtsZ2-1 (also known as FtsZ2 in A. thaliana) is more ancestral and contains a conserved carboxy (C)-terminal core motif similar to that of bacterial FtsZ. FtsZ2-2 (FtsZ1 in A. thaliana) lacks the $\mathrm{C}$-terminal core motif and is likely to be evolved by duplication of FtsZ2-1 or its precursor during plastid establishment after the endosymbiotic event (Miyagishima et al. 2004). However, the mechanisms of FtsZ-ring assembly and constriction in the PD machinery and the functional significance of the duplication and retention of two FtsZs during plastid evolution are not fully understood. In planta analysis of FtsZs is challenging because the FtsZ ring is incorporated into a complex with the rest of the PD machinery. Therefore, we investigated the assembly kinetics and contractile properties of the plastid FtsZ ring using the heterologous yeast system Pichia pastoris.

We expressed fluorescently tagged A. thaliana FtsZ2 (AF089738) and FtsZ1 (AY113896) with or without a C-terminal membrane-tethering sequence (MTS), which is originated from an amphipathic helix domain of Escherichia coli MinE, in the spherical cells of the yeast $P$. pastoris. As a result, MTS-tagged FtsZ2 assembled into a single, closed ring in Pichia cells and FtsZ1 without the MTS-tag also assembled into a ring (Figure $3 \mathrm{~A}$, upper set). Furthermore, we also succeeded to reconstitute the plastid FtsZ ring comprising both FtsZ2 and FtsZ1 interacted with the yeast cell membrane via $\mathrm{C}$ terminus of FtsZ2 (Figure $3 \mathrm{~A}$, bottom set). Taken together, we concluded that the reconstituted FtsZ ring in Pichia has a structure similar to that of the native FtsZ ring in plastid.

We next investigated the turnover kinetics of FtsZ2 and/or FtsZ1 in the single and co-assembled rings using fluorescence recovery after photobleaching (FRAP) assays to study the dynamics of ring assembly (Figure 3B). Protofilaments of tubulin are structurally similar to those of FtsZ at the steady state and are maintained by the turnover processes of polymerization/ depolymerization at both ends of the protofilaments. Owing to the similarities between FtsZ and tubulin, we surmised that the recovery of FtsZs in FRAP experiments depends on the dissociation rates ( $k_{\mathrm{offl}}$ and $k_{\mathrm{off} 2}$ ) determined by the depolymerization of the FtsZ protofilaments at both ends (Figure 
3C). Thus, we applied a two binding-state model to describe the turnover kinetics of polymerization/depolymerization at both ends of the FtsZ protofilaments (Figure 3D). Differences in the dissociation rates between $k_{\text {off1 }}$ and $k_{\text {off } 2}$ increased for both FtsZ2 and FtsZ1 in co-assembled rings as compared with those for rings assembled with just FtsZ2 or FtsZ1, indicating fast depolymerization at one end and much slower depolymerization at the other end of co-assembled filaments, and more similar depolymerization rates at both ends of filaments assembled by only FtsZ2 or FtsZ1 (Figure 3E). In addition, recovery of fluorescence intensity, which depends on the mobile fraction, of each FtsZ increased in the co-expression line compared with those in single-expression lines, which indicate increases in the numbers of FtsZ2 and FtsZ1 molecules that can polymerize and depolymerize in the co-assembled ring. Thus, this series of

A
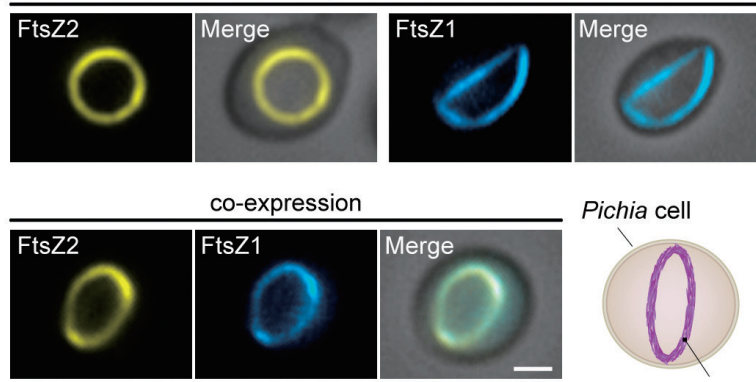

\section{Pichia cell}

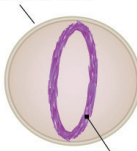

B

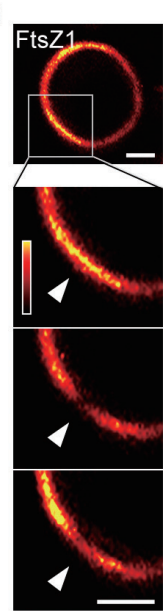

C

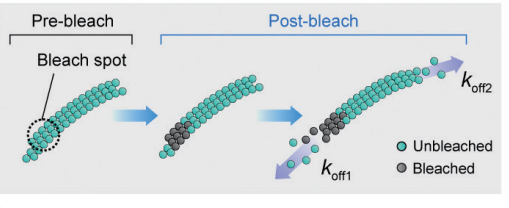

D $F R A P(t)=(1-r)\left(1-c_{\theta q 1} e^{-k_{\theta+1} t}-c_{\theta q 2} e^{-t_{\theta+2} t}\right)$
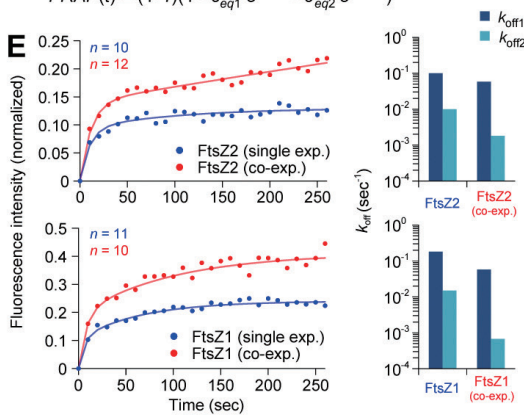

Figure 3 Reconstitution of a single FtsZ ring in spherical yeast Pichia pastoris cells. (A) Reconstituted FtsZ rings. Bar $=1 \mu \mathrm{m}$. (BE) Photobleaching experiments of fluorescent plastid FtsZs. FRAP experiments were carried out by bleaching of the fluorescent FtsZ2 or FtsZ1 at a part of the single ring to measure mobility of FtsZ molecules in the rings. Bar $=1 \mu \mathrm{m}$. Pre-bleached, bleached and recovery of a part of the FtsZ1 rings were shown as an example (B). Schematic representation of FRAP experiments of the reconstituted FtsZ ring (C). The best fitting curve was given by the two-binding-state model: $k_{\text {off } 1}$ and $k_{\text {off } 2}$ refer to dissociation rate constants, $C_{e q 1}$ and $C_{e q 2}$ refer to fractions of bound molecules and $r$ refer to the additional parameter for the effect of noncomplete recovery (D). Pre-bleached, bleached, and recovery of a part of the FtsZ rings were monitored for $270 \mathrm{~s}$ (E, left). Two dissociation rates $\left(k_{\mathrm{off} 1}\right.$ and $\left.k_{\mathrm{off} 2}\right)$ were computed from the recovery curves by the twoexponential model (E, right). Images were modified from Yoshida et al. (2016). experiments reveals that the coexistence of FtsZ2 and FtsZ1 may give rise to the polarity of polymerization of protofilament by stabilization one end of the protofilament.

The mechanism underlying the polarity of polymerization of the co-assembled protofilaments was revealed by chimeric versions of the FtsZ proteins. We designed two chimeric plastid FtsZ proteins, Z2-Z1 and Z1-Z2, in which the amino (N)-terminal halves of FtsZ1 and FtsZ2 were swapped to mimic the interfaces of putative heterodimers of FtsZ2 and FtsZ1 (Figure 4A). Fluorescence images of these chimeras in the Pichia cells showed that one filament (or occasionally two filaments) bundle was assembled (Figure 4B). The existence of filaments of Z2-Z1 or Z1-Z2 chimeras showed the hetero-interaction of both FtsZ2-FtsZ1 and FtsZ1-FtsZ2 by headto-tail cross-association of the individual GTP-binding domain and the GTPase-activating domain, indicating that FtsZ2 and FtsZ1 can assemble heteropolymers (Figure 4C). In addition, FRAP data showed that both Z2-Z1 and Z1-Z2 chimeras involved two distinct kinetic turnovers, one fast and one much slower dissociation reaction, similar to the FRAP data for the FtsZ2 and FtsZ1 co-assembled ring. Thus, these results suggest that heteropolymerization of FtsZ2 and FtsZ1 imparts polarity to polymerization of the co-assembled filament.

A

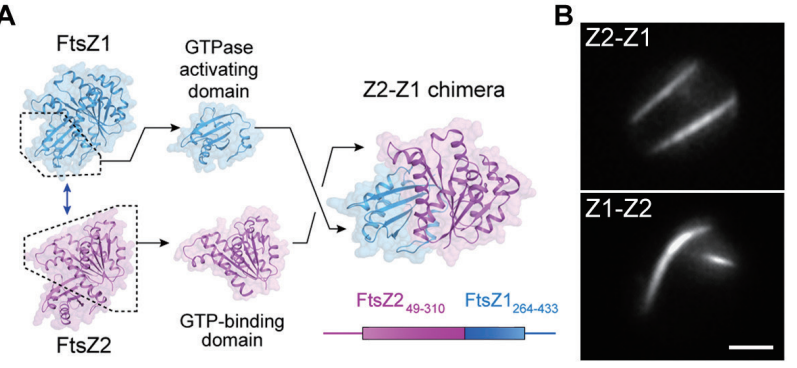

C

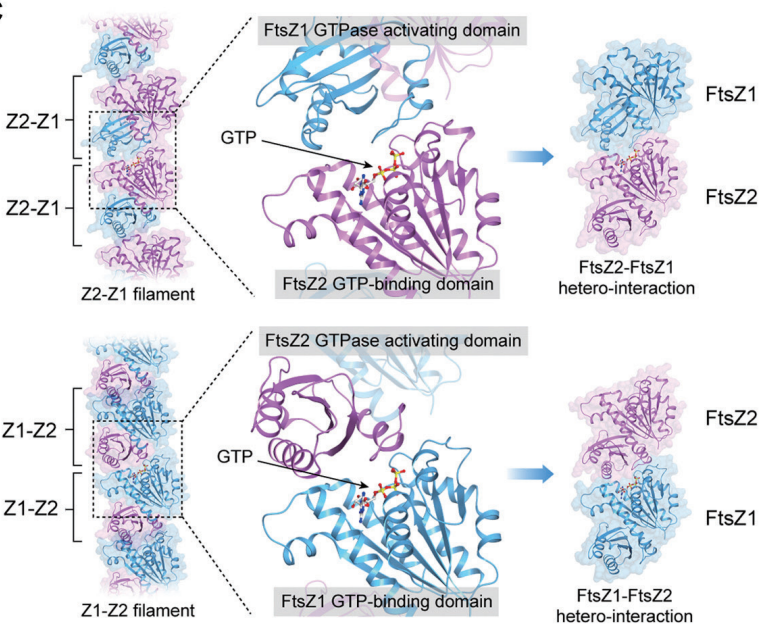

Figure 4 Hetero-interaction of plastid FtsZs. (A) A protein structure model of a chimeric-FtsZ named Z2-Z1 chimera. A protein structure of Z1-Z2 was omitted. (B) Protein distributions of Z2-Z1 chimera and Z1Z2 chimera. Bar $=1 \mu \mathrm{m}$. (C) Ribbon-type representations of heterointeractions of FtsZ2 and FtsZ1. FtsZ2 and FtsZ1 interact via GTPaseactivating domain and GTP-binding domain. Images were modified from Yoshida et al. (2016). 
Finally, we examined whether the FtsZ ring can generate a constrictive force required for fission of plastids. By acceleration of protein expression after the formation of the FtsZ rings, both the single- and co-assembled rings could constrict (Figure 5A). Interestingly, the co-assembled ring constricted more rapidly than the single-assembled ring comprising FtsZ2. Since the FtsZ1 ring did not constrict, the contraction of FtsZ rings may require membrane tethering. Cross-sectional images of the constricting FtsZ rings showed that the fluorescence intensity of FtsZ2 and/or FtsZ1 increased with the progress of contraction, suggesting that more FtsZ protofilaments accumulated in the ring (Figure 5B,

A

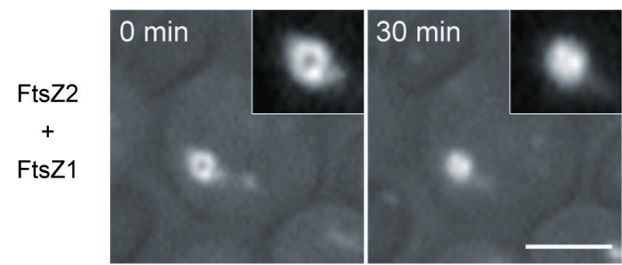

B

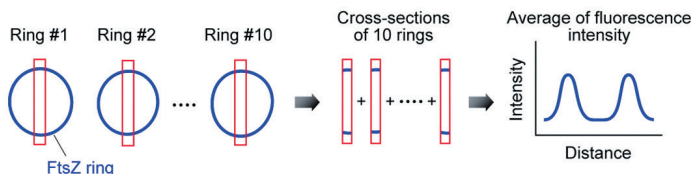

C

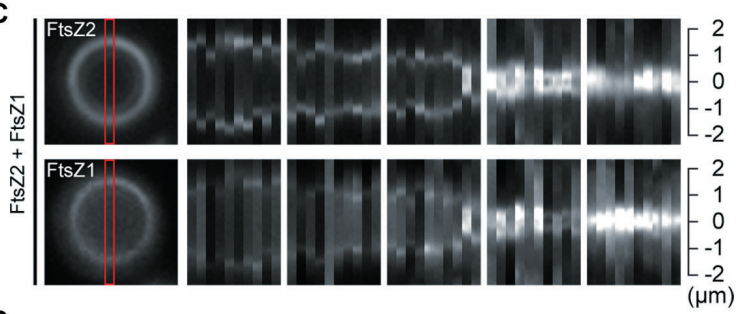

D

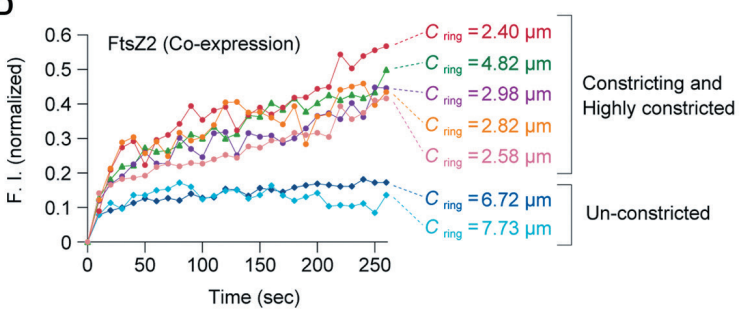

E

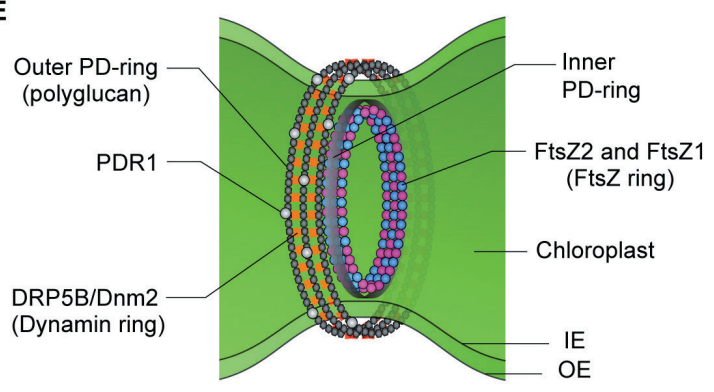

Figure 5 Constriction dynamics of the reconstituted plastid FtsZ rings. (A) Time-lapse images of the constricting ring comprising FtsZ2 and FtsZ1. Bar $=2 \mu \mathrm{m}$. (B and C) Fluorescence intensity profiles of the reconstituted plastid FtsZ rings during constriction. Schematic representations show a workflow of fluorescence intensity measurement of the rings (B). Ten cross-sectional images from individual rings in each time were aligned (C). (D) FRAP recovery of fluorescent FtsZ2 of the coassembled rings in un-constricting, constricting, and highly-constricted rings. (E) A schematic representation of structure of the PD machinery. Images were modified from Yoshida et al. (2016).
C). We then analyzed the turnover kinetics of the co-assembled rings in unconstricted, constricting and highly constricted rings. Unconstricted rings demonstrated similar FRAP curves at those observed previously (Figure 5D). In contrast, both constricting and highly constricted rings showed significant increase of fluorescence recovery after photobleaching. Although the molecular mechanism remains unclear, taking these results together, we propose that an increase in the amount of newly generated protofilaments within the ring induces a decrease in average protofilament length, increasing protofilament turnover and promoting contraction of the FtsZ ring (Yoshida et al. 2016).

\section{CONCLUSIONS AND REMARKS}

These studies shed light on the molecular mechanism of the PD machinery in plastid division as follows: the PD machinery is constructed from the inside rings, FtsZ and inner PD rings, to the outside rings, outer PD and dynamin rings (Figure 5E). The FtsZ ring is assembled by tubulin-like hetero-polymerization. After formation of the inside rings, the interaction of FtsZ and some membrane proteins might translate location information of the FtsZ ring to the outside of the plastid. Based on the information, the glycosyltransferase protein PDR1 likely binds to the outer membrane and biosynthesizes the polyglucan filaments to form the outer PD ring. Then, the GTPase protein dynamin (Dnm2) cross-links the outer PD ring filaments. After the assembly of the PD machinery, the plastid division site is constricted by both the FtsZ and PD-dynamin rings. The FtsZ ring generates the contractile force by increasing FtsZ protofilament turnover, and dynamin molecules crosslinking with the PD ring filaments generate the contractile force by the sliding-movement of the PD ring filaments. Taken together, these results indicate that the force for division of the plastid is generated and regulated from both inside and outside by dual contractible rings, the FtsZ ring and the PD-dynamin ring, as a supramolecular complex "the PD machinery". Here, we developed methods for isolation of the intact PD machineries from C. merolae cells and for the reconstitution of the plastid FtsZ ring in the yeast cells. These new extensible techniques will make it possible to further explore mechanism of plastid division at single-molecule level. Furthermore, we recently developed methods for isolation and multiple experimental system of the mitochondrial-dividing machinery from C. merolae cells (Yoshida et al. 2009, 2013). Interestingly, ultrastructural analysis and protein profiling of isolated MD machinery revealed significant similarities and differences between the mechanisms of division of plastids and mitochondria. As we have now obtained critically important techniques to decode molecular mechanisms of plastid and mitochondrial divisions, further investigations will also offer insights into how free-living cyanobacteria and $\alpha$-proteobacteria evolved into plastids and mitochondria during evolution.

\section{ACKNOWLEDGMENTS}

I gratefully acknowledge the contributions to the PD machinery research projects of past members of Kuroiwa laboratory (Rikkyo University), Kawano laboratory (The University of 
Tokyo) and to the reconstituted FtsZ ring project of past and present members of Osteryoung laboratory (Michigan State University). I am also grateful to the members of the selection committee for Encouragement Prize 2016 in the Japanese Society of Plant Morphology for their careful consideration. These works were supported by a Japan Society for the Promotion of Science Fellowships (No. 7488), a Japan Society for the Promotion of Science Postdoctoral Research Fellowship for Research Abroad and a Human Frontier Science Program Long Term Fellowship (No. LT000356/2011-L).

\section{REFERENCES}

Fujiwara, T., Misumi, O., Tashiro, K., Yoshida, Y., Nishida, K., Yagisawa, F., Imamura, S., Yoshida, M., Mori, T., Tanaka, K., Kuroiwa, H., and Kuroiwa, T. (2009) Periodic gene expression patterns during the highly synchronized cell nucleus and organelle division cycles in the unicellular red alga Cyanidioschyzon merolae. DNA Res 16: 59-72.

Fujiwara, T., Kanesaki, Y., Hirooka, S., Era, A., Sumiya, N., Yoshikawa, H., Tanaka, K., and Miyagishima, S. Y. (2015) A nitrogen source-dependent inducible and repressible gene expression system in the red alga Cyanidioschyzon merolae. Front Plant Sci 6: 1-10.

Fujiwara, T. (2017) Cytological and genomic features of the unicellular red alga Cyanidioschyzon merolae. Plant Morphol 29: 91-97 (in Japanese).

Gao, H., Kadirjan-Kalbach, D., Froehlich, J. E., and Osteryoung, K. W. (2003) ARC5, a cytosolic dynamin-like protein from plants, is part of the chloroplast division machinery. Proc Natl Acad Sci USA 100: 4328-4333.

Jarvis, P. and López-Juez, E. (2013) Biogenesis and homeostasis of chloroplasts and other plastids. Nat Rev Mol Cell Biol 14: 787-802.

Kuroiwa, T. (1998) The primitive red algae Cyanidium caldarium and Cyanidioschyzon merolae as model system for investigating the dividing apparatus of mitochondria and plastids. BioEssays 20: 344-354.

Kuroiwa, T., Kuroiwa, H., Sakai, A., Takahashi, H., Toda, K., and Itoh, R. (1998) The division apparatus of plastids and mitochondria. Int Rev Cytol 181: 1-41.

Kuroiwa, T., Misumi, O., Nishida, K., Yagisawa, F., Yoshida, Y., Fujiwara, T., and Kuroiwa, H. (2008) Vesicle, mitochondrial, and plastid division machineries with emphasis on dynamin and electron-dense rings. Int Rev Cell Mol Biol 271: 97-152.

Matsuzaki, M., Misumi, O., Shin-I, T., Maruyama, S., Takahara, M., Miyagishima, S. Y., Mori, T., Nishida, K., Yagisawa, F., Nishida, K., Yoshida, Y., Nishimura, Y., Nakao, S., Kobayashi, T., Momoyama, Y., Higashiyama, T., Minoda, A., Sano, M., Nomoto, H., Oishi, K., Hayashi, H., Ohta, F., Nishizaka, S., Haga, S., Miura, S., Morishita, T., Kabeya, Y., Terasawa, K., Suzuki, Y., Ishii, Y., Asakawa, S., Takano, H., Ohta, N., Kuroiwa, H., Tanaka, K., Shimizu, N., Sugano, S., Sato, N., Nozaki, H., Ogasawara, N., Kohara, Y., and Kuroiwa, T. (2004) Genome sequence of the ultrasmall unicellular red alga Cyanidioschyzon merolae 10D. Nature
428: 653-657.

Minoda, A., Sakagami, R., Yagisawa, F., Kuroiwa, T., and Tanaka, K. (2004) Improvement of culture conditions and evidence for nuclear transformation by homologous recombination in a red alga, Cyanidioschyzon merolae 10D. Plant Cell Physiol 45: 667-671.

Misumi, O., Matsuzaki, M., Nozaki, H., Miyagishima, S. Y., Mori, T., Nishida, K., Yagisawa, F., Yoshida, Y., Kuroiwa, H., and Kuroiwa, T. (2005) Cyanidioschyzon merolae genome. A tool for facilitating comparable studies on organelle biogenesis in photosynthetic eukaryotes. Plant Physiol 137: 567-585.

Miyagishima, S. Y., Takahara, M., and Kuroiwa, T. (2001) Novel filaments $5 \mathrm{~nm}$ in diameter constitute the cytosolic ring of the plastid division apparatus. Plant Cell 13: 707-721.

Miyagishima, S. Y., Nishida, K., Mori, T., Matsuzaki, M., Higashiyama, T., Kuroiwa, H., and Kuroiwa, T. (2003) A plant-specific dynamin-related protein forms a ring at the chloroplast division site. Plant Cell 15: 655-665.

Miyagishima, S. Y., Nozaki, H., Nishida, K., Nishida, K., Matsuzaki, M., and Kuroiwa, T. (2004) Two types of FtsZ proteins in mitochondria and red-lineage chloroplasts: the duplication of FtsZ is implicated in endosymbiosis. $J$ Mol Evol 58: 291-303.

Miyagishima, S. Y., Nakanishi, H., and Kabeya, Y. (2011) Structure, regulation, and evolution of the plastid division machinery. Int Rev Cell Mol Biol 291: 115-153.

Mori, T., Kuroiwa, H., Takahara, M., Miyagishima, S. Y., and Kuroiwa, T. (2001) Visualization of an FtsZ ring in chloroplasts of Lilium longiflorum leaves. Plant Cell Physiol 42: 555-559.

Nozaki, H., Takano, H., Misumi, O., Terasawa, K., Matsuzaki, M., Maruyama, S., Nishida, K., Yagisawa, F., Yoshida, Y., Fujiwara T, Takio, S., Tamura, K., Chung, S. J., Nakamura, S., Kuroiwa, H., Tanaka, K., Sato, N., and Kuroiwa, T. (2007) A 100\%-complete sequence reveals unusually simple genomic features in the hot-spring red alga Cyanidioschyzon merolae. BMC Biol 5: 28.

Ohnuma, M., Misumi, O., Fujiwara, T., Watanabe, S., Tanaka, K., and Kuroiwa, T. (2009) Transient gene suppression in a red alga, Cyanidioschyzon merolae 10D. Protoplasma 236: 107-112.

Osteryoung, K. W. and Pyke, K. A. (2014) Division and dynamic morphology of plastids. Annu Rev Plant Biol 65: 443-72.

Sumiya, N., Fujiwara, T., Kobayashi, Y., Misumi, O., and Miyagishima, S. Y. (2014) Development of a heatshock inducible gene expression system in the red alga Cyanidioschyzon merolae. PLoS One 9: 1-11.

TerBush, A. D., Yoshida, Y, and Osteryoung, K. W. (2013) FtsZ in chloroplast division: structure, function and evolution. Curr Opin Cell Biol 25: 461-470.

Vitha, S., McAndrew, R. S., and Osteryoung, K. W. (2001) FtsZ ring formation at the chloroplast division site in plants. $J$ Cell Biol 153: 111-120.

Yoshida, M., Yoshida, Y., Fujiwara, T., Misumi, O., Kuroiwa, 
H., and Kuroiwa, T. (2011) Proteomic comparison between interphase and metaphase of isolated chloroplasts of Cyanidioschyzon merolae (Cyanidiophyceae, Rhodophyta). Phycol Res 59: 1-15.

Yoshida, Y., Kuroiwa, H., Misumi, O., Nishida, K., Yagisawa, F., Fujiwara, T., Nanamiya, H., Kawamura, F., and Kuroiwa, T. (2006) Isolated chloroplast division machinery can actively constrict after stretching. Science 313: 1435-1438.

Yoshida, Y., Kuroiwa, H., Hirooka, S., Fujiwara, T., Ohnuma, M., Yoshida, M., Misumi, O., Kawano, S., and Kuroiwa, T. (2009) The bacterial ZapA-like protein ZED is required for mitochondrial division. Curr Biol 19: 1491-1497.

Yoshida, Y., Kuroiwa, H., Misumi, O., Yoshida, M., Ohnuma, M., Fujiwara, T., Yagisawa, F., Hirooka, S., Imoto, Y., Matsushita, K., Kawano, S., and Kuroiwa, T. (2010) Chloroplasts divide by contraction of a bundle of nanofilaments consisting of polyglucan. Science 329: 949953.

Yoshida, Y., Miyagishima, S. Y., Kuroiwa, H., and Kuroiwa, T. (2012) The plastid-dividing machinery: formation, constriction and fission. Curr Opin Plant Biol 15: 714-721.

Yoshida, Y., Fujiwara, T., Imoto, Y., Yoshida, M., Ohnuma, M., Hirooka, S., Misumi, O., Kuroiwa, H., Kato, S., Matsunaga S., and Kuroiwa, T. (2013) The kinesin-like protein TOP promotes Aurora localisation and induces mitochondrial, chloroplast and nuclear division. J Cell Sci 126: 2392-2400.

Yoshida, Y., Mogi, Y., TerBush, A. D., and Osteryoung, K. W. (2016) Chloroplast FtsZ assembles into a contractible ring via tubulin-like heteropolymerization. Nat Plants 2: 16095.

Yoshida, Y. and Mogi, Y. (2016) Reconstitution of the chloroplast FtsZ ring using the heterologous yeast system Pichia pastoris. Cytologia 81: 249-250.

Received: 16 December 2016 / Accepted: 21 January 2017 